\title{
Les stratégies de commande appliquées au contrôle d'écoulement : État de l'art
}

\author{
Mathieu Rouméas ${ }^{1}$, Patrick Gilliéron ${ }^{1}$ et Azeddine Kourta Ka $^{2, a}$ \\ 1 Direction de la Recherche Renault SAS, Service 64260, code API : TCR AVA 058, 1 Av. du Golf, 78288 Guyancourt Cedex, \\ France \\ 2 Institut de Mécanique des Fluides de Toulouse, Allée du professeur Camille Soula, 31000 Toulouse, France
}

Reçu le 27 février 2004, accepté le 3 Avril 2007

\begin{abstract}
Résumé - Le contrôle des écoulements est à la base des travaux de développement en aérodynamique automobile. L'objectif à moyen terme est d'obtenir des systèmes de contrôle réactif où l'actionneur agit en fonction de l'état de l'écoulement. Ce type de contrôle implique la mise en place d'un actionneur, d'un capteur et d'une loi de commande les reliant l'un à l'autre. Cet article constitue un état de l'art sur les stratégies de commande envisageables. Il propose un classement théorique des différents schémas utilisés dans la littérature. Les commandes en boucles fermées sont plus particulièrement développées. Elles peuvent se décliner autour de 2 types de schémas : les boucles fermées simples où des mesures de l'écoulement prises en aval de l'actionneur sont utilisées dans la loi de commande ou des boucles fermées de type prédicteur/correcteur comme les schémas adaptatifs ou les schémas issus de la théorie du contrôle optimal. Pour chaque schéma de contrôle, les concepts mathématiques et algorithmiques sont évoqués et des résultats bibliographiques sont présentés. La pertinence de ces schémas pour le secteur automobile est ensuite discutée.
\end{abstract}

Mots clés : Contrôle actif / simulation numérique / réduction de traînée / contrôle en boucle ouverte / contrôle en boucle fermée / contrôle adaptatif / contrôle nominal

\begin{abstract}
Flow control strategy: state of the art. Flow control is the basis for most automotive aerodynamics developments. A mid-term objective is to obtain active-control systems for which the actuator reacts to the state of the flow. This type of control requires the set up of an actuator, a sensor and a command law connecting them. This paper reviews the state of the art for the possible command strategies. A theoretical sorting of the different schemes found in the literature is proposed. Closed-loop commands are specifically developed. They can be organized into two kinds of schemes: simple closed loops with a command law using flow measurements performed downstream the actuator, or closed loops of the predictor/corrector type such as adaptive schemes, or sub-optimal schemes. For each of them, mathematical and algorithmical concepts are presented, and a literature review is performed. The relevance of these schemes for automotive applications is finally discussed.
\end{abstract}

Key words: Active control / numerical simulation / drag reduction / feedforward control / feedback control / adaptative control / optimal control

\section{Introduction}

Dans tous les domaines liés à la dynamique des fluides, notamment l'aéronautique et l'automobile, le contrôle de l'écoulement est devenu incontournable pour améliorer les performances, la sécurité et le confort. Les décollements et les structures tourbillonnaires qu'ils génèrent entraînent en effet une détérioration des performances

\footnotetext{
${ }^{a}$ Auteur correspondant : kourta@imft.fr
}

aérodynamiques, avec notamment une augmentation de la consommation directement liée à l'augmentation de la traînée aérodynamique. Par ailleurs, la formation de structures tourbillonnaires organisées est généralement néfaste pour les concepteurs automobiles en terme de prestations acoustiques ou de stabilité aérodynamique.

Ces dix dernières années, les études se sont ainsi orientées vers un contrôle actif où la quantité de mouvement de l'écoulement est modifiée par un apport en 


\section{Nomenclature}

\begin{tabular}{|lll|}
\hline $\boldsymbol{V}$ & Vecteur vitesse locale & $\mathrm{m} . \mathrm{s}^{-1}$ \\
$u_{1}$ & Composante sur l'axe $x_{1}$ de la vitesse $\boldsymbol{V}$ & $\mathrm{m} . \mathrm{s}^{-1}$ \\
$u_{2}$ & Composante sur l'axe $x_{2}$ de la vitesse $\boldsymbol{V}$ & $\mathrm{m} . \mathrm{s}^{-1}$ \\
$u_{3}$ & Composante sur l'axe $x_{3}$ de la vitesse $\boldsymbol{V}$ & $\mathrm{m} . \mathrm{s}^{-1}$ \\
$U_{\mathrm{e}}$ & Vitesse infinie amont & $\mathrm{m} . \mathrm{s}^{-1}$ \\
$p$ & Pression statique & $\mathrm{Pa}$ \\
$p^{\prime}$ & Fluctuation de pression & $\mathrm{Pa}$ \\
$p_{\mathrm{e}}$ & Pression infinie amont & $\mathrm{Pa}$ \\
$p^{\text {id }}$ & Pression idéal (gaz parfait) & $\mathrm{Pa}$ \\
$C_{\mathrm{r}}$ & Coefficient de récupération de pression & \\
$\boldsymbol{q}$ & Vecteur d'état & $\mathrm{m} . \mathrm{s}^{-1}$ \\
$\phi$ & Amplitude du contrôle & $\mathrm{m} . \mathrm{s}^{-1}$ \\
$\eta$ & Vitesse adjointe & $\mathrm{Pa}$ \\
$\pi$ & Pression adjointe & $\mathrm{m}^{2}$ \\
$\Gamma_{\mathrm{c}}$ & Surface de contrôle & $\mathrm{m}^{2}$ \\
$\Gamma_{\mathrm{s}}$ & Surface de prise de mesure & \\
\hline
\end{tabular}

énergie. L'intérêt de tels systèmes réside dans la possibilité de développer des actionneurs réactifs, des systèmes prenant en compte la réaction de l'écoulement.

Le contrôle actif, ou réactif, passe par la définition de trois paramètres : un capteur qui mesure des variables physiques de l'écoulement et teste l'effet du contrôle, un actionneur qui apporte de l'énergie à l'écoulement et une loi de commande qui permet d'adapter la réponse de l'actionneur aux variables physiques de l'écoulement. Cette synthèse s'intéresse au troisième point, à savoir la définition d'une stratégie de commande. Dans la première partie, un état de l'art sur les différentes possibilités de simulation numérique du contrôle actif est présenté. Loin d'être exhaustif, il a cependant la prétention de proposer un large spectre de l'état actuel des connaissances sur le sujet. Pour chaque schéma de contrôle, les résultats sont présentés. La seconde partie s'intéresse plus en détail aux schémas en boucles fermées et les procédures de mise en place sont détaillées à travers des exemples tirés de la bibliographie. Des schémas de simplification couramment utilisés sont présentés dans la troisième partie. Enfin, la dernière partie propose une discussion sur l'application de ces différentes stratégies au problème de réduction de traînée sur un véhicule automobile.

\section{Différents types de contrôle}

Plusieurs stratégies sont envisageables pour définir la loi de commande : la nature de l'écoulement à contrôler, l'objectif du contrôle en terme de performance, les moyens de calcul ou les critères de temps de réaction sont autant de facteurs qui déterminent le choix de cette stratégie. Gad-El-Hak [1] et Moin \& Bewley [2] proposent un classement de ces différentes stratégies (Fig. 1). Le contrôle peut ainsi être actif ou passif selon qu'il apporte de l'énergie ou non à l'écoulement. Le contrôle passif est forcément prédéterminé puisque les caractéristiques du

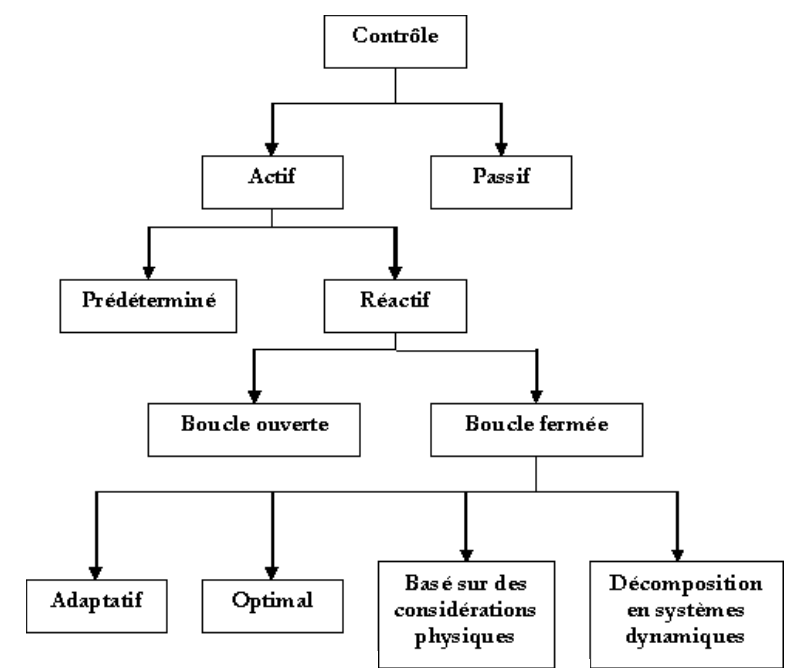

Fig. 1. Classement des différents types de contrôle, d'après Gad-El-Hak [1].

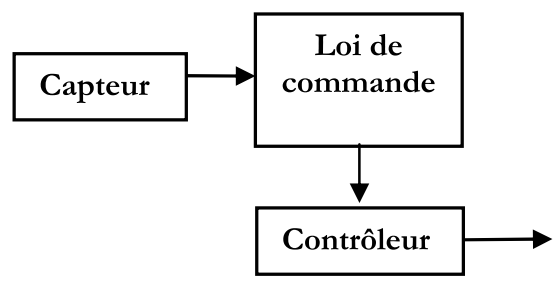

Fig. 2. Schéma de contrôle en boucle ouverte.

contrôle n'évoluent pas au cours du temps. Le contrôle actif peut au contraire être commandé et permettre un contrôle réactif grâce à des commandes en boucle ouverte ou fermée. Ce classement reste cependant très théorique et pédagogique. En pratique, la mise en place d'un contrôle nécessite généralement une solution hybride [3].

Le contrôle prédéterminé impose une loi de commande à l'actionneur indépendamment de la réaction du système. Il ne nécessite aucun capteur et est donc très facile à mettre en place. Du fait de sa simplicité, il est couramment utilisé dans la littérature, mais ne permet pas d'envisager un contrôle réactif. Dans le contrôle en boucle ouverte, au contraire la perturbation apportée par l'actionneur est pilotée par l'état de l'écoulement. Un capteur placé en amont de l'actionneur renseigne ce dernier sur les caractéristiques de l'écoulement infini amont. La perturbation apportée à l'écoulement est alors directement liée à l'état du système en amont de l'actionneur. Une prise de pression peut alors par exemple servir de signal d'entrée selon le schéma présenté figure 2 .

Cette étude porte plus spécifiquement sur les stratégies de contrôle réactives qui tiennent compte de l'évolution du système. Le but est de définir les paramètres de l'actionneur en fonction de ceux de l'écoulement, mais également en fonction de la réaction de l'écoulement au contrôle, afin d'optimiser les performances du contrôleur. En ce sens, cet article approfondit la notion de boucle fermée. 


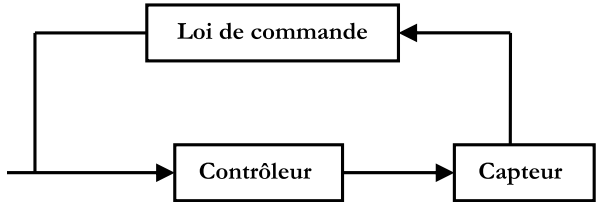

Fig. 3. Schéma de contrôle en boucle fermée.

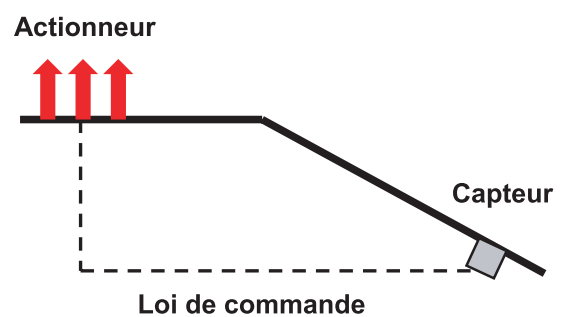

Fig. 4. Contrôle par un jet d'après Creusé [4].

\section{Contrôle en boucle fermée}

\section{Boucle fermée simple}

La première solution de commande en boucle fermée consiste à utiliser dans la loi de commande de l'actionneur une variable caractéristique de l'écoulement mesurée en aval de la position de l'actionneur (Fig. 3). Cette variable est représentative de l'écoulement et de la réaction de l'écoulement au contrôle.

Dans une étude portant sur la maximisation de la poussée d'un écoulement laminaire sur un dièdre, Creusé [4] a ainsi mis en place une fonction de transfert constituée d'un déphaseur et d'un amplificateur de puissance. Le schéma de la boucle de commande est du type de celui présenté figure 3. L'écoulement se fait autour d'un dièdre d'angle $\alpha=10^{\circ}$ pour un nombre de Mach incident de 0,2 et un nombre de Reynolds $R e=400$ défini à partir de la vitesse infinie amont et de l'épaisseur de couche limite $\delta$. Un jet injecte du fluide dans l'écoulement avec une vitesse d'injection définie par une loi de commande en boucle fermée (Fig. 4). La vitesse d'injection varie linéairement par rapport au gradient de pression, la pente étant fixée par le rapport de la vitesse sur le gradient de pression relevé pour un gradient de pression maximum.

$$
\frac{v(t)}{\left(\frac{\partial p}{\partial l}\right)^{\prime}}=\frac{\varepsilon}{\left(\frac{\partial p}{\partial l}\right)_{\max }^{\prime}}
$$

où le prime indique la dérivée temporelle, $\partial l$ un déplacement élémentaire tangent à la paroi, $\varepsilon$ la vitesse lorsque le gradient de pression longitudinal est maximal. Le but est de limiter l'amplitude des structures tourbillonnaires issues du décollement localisé sur la rupture de pente. La performance du contrôle est donnée par un coefficient de récupération de pression défini par [4] :

$$
C_{\mathrm{r}}=\frac{T_{\mathrm{p}}^{\mathrm{num}}-\left(T_{\mathrm{p}}^{\mathrm{id}}\right)_{1}}{\left(T_{\mathrm{p}}^{\mathrm{id}}\right)_{2}}
$$

Le terme $T_{\mathrm{p}}^{\text {num }}$ représente l'intégrale de la pression sur la partie en aval de la rupture de pente, calculée

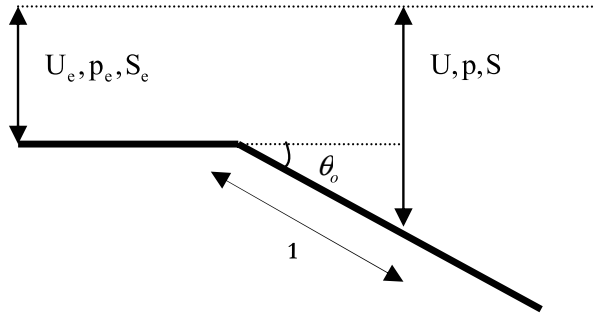

Fig. 5. Contrôle par un jet, d'après Creusé [4].

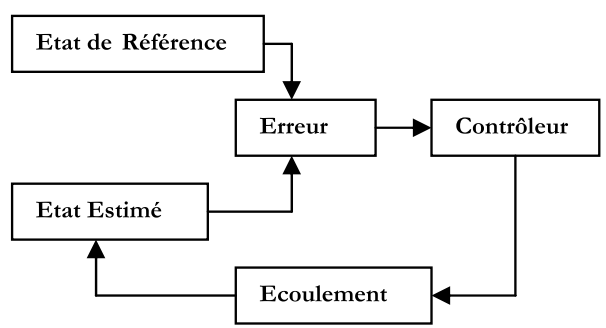

Fig. 6. Schéma de contrôle en boucle fermée.

numériquement, avec $\boldsymbol{x}$ le vecteur directeur de la direction longitudinale et $\boldsymbol{n}$ le vecteur normal à la paroi. L'effort dû à la pression atmosphérique est donné $\operatorname{par}\left(T_{\mathrm{p}}^{\mathrm{id}}\right)_{1}=L p_{\mathrm{e}}$ où $L$ représente la longueur de la partie aval du dièdre et $p_{\mathrm{e}}$ la pression en amont de la cassure du dièdre, égale à la moyenne temporelle de la pression en entrée du domaine de calcul, évaluée au cours de la simulation. Enfin, l'effort induit par l'écoulement est donné par [4] :

$$
\left(T_{\mathrm{p}}^{\mathrm{id}}\right)_{2}=L \frac{\rho U_{\mathrm{e}}^{2}}{2}\left(1-\frac{1}{1+\frac{L \sin \theta_{\mathrm{o}}}{S_{\mathrm{e}}}}\right)
$$

avec $U_{\mathrm{e}}$ la vitesse infinie amont, $S_{\mathrm{e}}$ la section d'entrée, et $\theta_{\mathrm{o}}=-10^{\circ}$ (Fig. 5). Pour un écoulement non contrôlé, le coefficient de récupération de pression $C_{\mathrm{r}}=64,61 \%$. Lorsque le contrôle en boucle ouverte est appliqué, il devient égal à $84,08 \%$.

\section{Schéma prédicteur/correcteur}

Dans la deuxième solution, de type prédicteur correcteur (Fig. 6), la variable mesurée est retournée au contrôleur afin de la comparer à une donnée de référence cible. Un algorithme est alors mis en place dans le but de minimiser l'erreur ainsi définie. La stratégie employée peut alors se moduler autour de deux types de schémas qui se distinguent par leur dépendance mathématique aux équations gouvernant la dynamique de l'écoulement à contrôler [2] : les schémas adaptatifs qui utilisent des algorithmes intelligents sans connaissance a priori de l'écoulement et les schémas basés sur la théorie du contrôle optimal où une fonctionnelle de coût est à minimiser. 


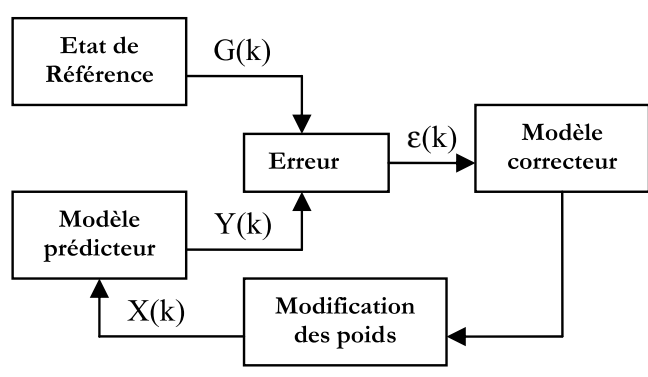

Fig. 7. Schéma de contrôle adaptatif.

\subsection{Schéma de contrôle adaptatif}

\subsubsection{Idée générale}

La stratégie de contrôle adaptatif utilise un algorithme permettant de modéliser l'écoulement sans connaissance a priori de celui-ci. L'action d'un contrôleur adaptatif évolue au cours du temps, de façon à ce que son comportement s'ajuste à une performance attendue.

Le système de contrôle ne tient aucun compte de la physique de l'écoulement ou des équations qui gouvernent son évolution, mais se contente de modifier empiriquement les paramètres du contrôle afin d'atteindre un état cible de référence. Cette connaissance empirique du système passe par une loi d'apprentissage qui indique au système de contrôle l'influence de ses actions précédentes sur l'écoulement. La procédure de contrôle adaptatif peut ainsi être représentée par un modèle de type prédicteur/correcteur (Fig. 7).

Le processus est itératif et indexé par un entier $k$. $X(k)$ est un vecteur d'état qui représente typiquement le contrôle appliqué à l'écoulement à l'itération $k$. Le vecteur de sortie $Y(k)$ constitue une estimation du paramètre à contrôler; c'est par exemple le gradient tangent de pression localisée au voisinage de l'arête d'un dièdre dans le cas de Creusé [4]. L'état de référence $G(k)$ est comparé au vecteur de sortie $Y(k)$, ce qui définit l'erreur $\varepsilon^{k}$.

Le modèle prédicteur va pondérer le vecteur d'état de façon à estimer au mieux la dynamique du système. Cette pondération est ensuite modifiée par le correcteur à travers un processus adaptatif tel que celui proposé par Creusé [4], présenté ici à titre d'exemple.

\subsubsection{Procédure de mise en place}

L'objectif est que la répartition des pressions tende vers un état idéal défini par l'écoulement en fluide parfait autour du dièdre, caractérisé par une répartition idéale de pression $p^{\text {id }}$.

La première étape identifie l'écoulement. Soit $G^{k}$ le gradient tangent de pression en écoulement fluide parfait au niveau du capteur (Fig. 3) à l'instant $t=k \Delta t$ où $\Delta t$ représente le pas de temps de la simulation, $Y^{k}$ une estimation du gradient tangent de pression en écoulement réel, l'objectif est de faire tendre $Y^{k}$ vers $G^{k}$, soit

$$
\left(Y^{k}-G^{k}\right) \underset{k \rightarrow \infty}{\longrightarrow} 0
$$

Le vecteur d'état de l'actionneur $Q^{k}$ et le vecteur des poids $W^{k}$ à l'itération $k$ sont également définis par :

$$
\begin{aligned}
& Q^{k}=\left[q^{k-r-1}, q^{k-r-2}, \ldots, q^{k-r-n}\right] \\
& W^{k}=\left[w_{k}^{1}, w_{k}^{2}, \ldots, w_{k}^{n p}\right]
\end{aligned}
$$

où $n p$ est le nombre de poids du système d'identification, avec $q^{k}$ l'amplitude de la vitesse du fluide injecté par l'actionneur au temps $t=k \Delta t, w_{k}^{i}$ le poids représentant l'influence du soufflage effectué au temps $t=(k-i-r) \Delta t$ sur l'écoulement au temps $t=k \Delta t, r$ étant un paramètre de retard lié au temps de réponse du système d'identification.

L'écoulement est alors identifié comme :

$$
Y^{k}=\sum_{i=1}^{n p} w_{k}^{i} q^{k-r-i}
$$

Le processus adaptatif va ainsi prendre en compte les perturbations apportées à l'écoulement aux itérations passées et la façon dont elles ont influencé la dynamique de l'écoulement. La deuxième étape, de correction, cherche à minimiser l'écart entre l'écoulement réel et l'état cible. Cette optimisation passe par la modification de la matrice de poids $W^{k}$. Soit $\varepsilon^{k}$ l'erreur :

$$
\varepsilon_{k}=G^{k}-Y^{k}=G^{k}-\left(W^{k}\right)^{T} Q^{k}
$$

avec $\left(W^{k}\right)^{T}$ le transposé du vecteur poids.

$$
\begin{aligned}
\varepsilon_{k}^{2}= & \left(G^{k}\right)^{2}-2 G^{k}\left(W_{k}^{2} Q^{k}\right)+\left(W^{k}\right)^{T} Q^{k}\left(Q^{k}\right)^{T} W_{k} \\
E\left[\varepsilon_{k}^{2}\right]= & E\left[\left(G^{k}\right)^{2}\right]+\left(W^{k}\right)^{T} E\left[Q^{k}\left(Q^{k}\right)^{T}\right] W_{k} \\
& -2 E\left[G^{k}\left(Q^{k}\right)^{T}\right] W_{k}
\end{aligned}
$$

où l'opérateur $E[-]$ représente l'espérance mathématique. Cette expression devient alors :

$$
\alpha=E\left[\left(G^{k}\right)^{2}\right]+\left(W^{k}\right)^{T} R W_{k}-2 P^{T} W_{k}
$$

où $\alpha$ est une fonction quadratique des composantes de la matrice de poids, $R$ représente l'autocorrélation du signal d'entrée et $P$ l'intercorrélation entre la réponse désirée et l'entrée. La matrice de poids optimale qui minimise le carré de l'erreur $\varepsilon^{k}$ annule le gradient de $\alpha$ :

$$
\left\{\begin{array}{l}
\nabla=\frac{\partial \alpha}{\partial W}=2 R W-2 P \\
W *=R^{-1} P
\end{array}\right.
$$

La détermination de $W *$ passe par l'inversion d'une matrice, ce qui est généralement l'opération limitante en terme de temps de réaction. Afin de limiter le coût de calcul, $W *$ sera calculée par un algorithme adaptatif ne nécessitant pas le calcul du gradient (5). L'algorithme retenu est celui de Last-Mean-Square (LMS) où le carré de l'erreur est minimisé [5]. Pour alléger les calculs, $\alpha$ est approchée par $\alpha_{k}=\left(\varepsilon^{k}\right)^{2}$ ce qui permet de définir une 
erreur instantanée, sans passer par la moyenne. Ainsi, le processus sera optimisé à chaque itération :

$$
\nabla_{k}=\frac{\partial \alpha_{k}}{\partial W_{k}}=2 \varepsilon_{k} \frac{\partial \varepsilon_{k}}{\partial W_{k}}=-2 \varepsilon_{k} Q^{k}
$$

$\mathrm{Au}$ moyen de cette estimation du gradient, le vecteur poids à l'itération $k+1$ est défini par un algorithme de type plus profonde descente [5] :

$$
W_{k+1}=W_{k}-\mu \nabla_{k}=W_{k}+2 \varepsilon_{k} \mu Q^{k}
$$

où $\mu$ est un gain constant qui régule la stabilité et la vitesse du processus.

L'équation (7) représente la loi d'apprentissage qui est le fondement de la stratégie adaptative. Le processus est optimisé sans connaissance a priori du système, mais en fonction des itérations précédentes puisque le vecteur $Q^{k}$ contient le forçage des itérations passées. C'est un système à mémoire qui apprend et peut s'adapter.

Il reste alors à exprimer explicitement le vecteur de contrôle $Q^{k}$ à appliquer au système. L'expression (3) peut se réécrire sous la forme :

$$
Y^{k+r+1}=\sum_{i=1}^{n p} w_{k+r+1}^{i} q^{k-i+1}
$$

La connaissance des valeurs du contrôle aux itérations précédentes, à savoir les $q^{k-i+1}, i=1 \ldots n p$, permet de déterminer le contrôle à appliquer à $t=k . \Delta t$ à l'écoulement afin d'obtenir au pas de temps suivant le vecteur cible $G^{k+r+1}$, soit :

$$
\begin{gathered}
Y^{k+r+1}=G^{k+r+1} \\
q^{k}=\frac{G^{k+r+1}-\sum_{i=2}^{n p} w_{i}^{k+r+1} q^{k-i+1}}{w_{1}^{k+r+1}}
\end{gathered}
$$

En considérant que l'adaptation des poids est assez lente, l'équation (10) donne :

$$
q^{k}=\frac{G^{k+r+1}-\sum_{i=2}^{n p} w_{k}^{i} q^{k-i+1}}{w_{k}^{1}}
$$

La valeur du contrôle à appliquer au temps $t=k \Delta t$ est donc déterminée en fonction de l'estimation de l'état de l'écoulement et des perturbations apportées aux itérations précédentes.

La procédure peut se résumer comme suit : l'état cible $G^{k}$ est connu pour tout $k$. Il doit être déterminé par une simulation en écoulement fluide parfait où le gradient tangent de pression est relevé. À l'itération $\mathrm{k}$, le vecteur d'état de l'actionneur au temps $t=(k-$ 1) $\Delta t Q^{k-1}$ et le vecteur poids $W^{k}$ représentatif de l'état de l'écoulement sont connus. L'expression (11) permet alors de déterminer $Q^{k}$. La relation (4) définit l'erreur $\varepsilon^{k}$ et $W^{k+1}$ est déterminé par (7) en minimisant le carré de l'erreur à chaque itération.

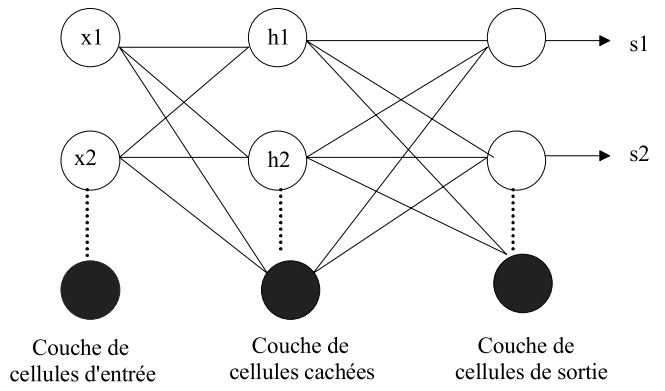

Fig. 8. Schéma d'un réseau multicouche.

Une stratégie de contrôle adaptatif appliquée à l'exemple de Creusé [4] permet d'augmenter de façon significative le coefficient de récupération de pression $\mathrm{Cr}$ défini en 2.1 par l'expression (2). Il passe de $64 \%$ en écoulement non contrôlé à 84,71\%.

Pour des configurations où l'écoulement moyen en amont du véhicule varie en intensité et en direction, comme c'est souvent le cas en automobile (phase de dépassement, vent latéral, ...), il est néanmoins intéressant de développer un contrôleur qui soit réactif et capable de s'adapter. Un tel contrôleur nécessite de nombreux capteurs, afin de pouvoir modéliser, identifier l'écoulement de façon efficace. Le problème qui se pose alors est celui du coût en calcul. Un trop grand temps de réponse rend en effet caduque la réactivité du système. Dans cette optique, les réseaux de neurones, qui permettent une utilisation efficace du calcul parallèle, peuvent constituer une alternative intéressante comme le laissent supposer les premiers résultats bibliographiques [6].

\subsubsection{Réseau de neurones}

Le réseau de neurones est un algorithme d'apprentissage qui doit choisir à l'intérieur d'une classe de modèles celui correspondant le mieux à une distribution de données d'entrée [7,8]. L'algorithme peut se décomposer en 2 phases : une phase d'activation et une phase d'apprentissage.

Le réseau est organisé en couches. La couche d'entrée transmet ses résultats à la couche supérieure, qui retransmet ses données résultantes à la suivante et ainsi de suite jusqu'à la couche de sortie qui fournit le neurone de sortie [6-8]. Les couches intermédiaires sont appelées couches cachées (Fig. 8). Chaque couche est constituée d'une ou plusieurs unités qui reçoivent de nombreuses entrées qu'elles combinent sous la forme d'une valeur de sortie unique. Cette combinaison est appelée la fonction d'activation de l'unité qui se décline en une fonction de combinaison et une fonction de transfert. Dans l'exemple étudié, la fonction de combinaison est une somme pondérée des signaux d'entrée, une fonction linéaire, et la fonction de transfert une fonction non-linéaire en hyperbolique tangente $[6]$.

La phase d'apprentissage permet au réseau d'évoluer dans le temps en tenant compte des expériences antérieures. L'importance relative des différentes connexions 
est modifiée en fonction des résultats précédents afin de trouver le meilleur modèle par rapport aux exemples donnés. L'apprentissage par rétro propagation consiste à descendre le réseau de façon itérative en ajustant les poids à chaque passage selon le calcul d'erreur. Pour cela, un algorithme de rétro propagation de l'erreur est mis en œuvre. La matrice des poids est d'abord initialisée au hasard. Un exemple est choisi et appliqué en entrée. Le calcul de cette entrée est ensuite propagé dans le réseau pour obtenir la sortie correspondante, qui est comparée à la sortie désirée. La contribution d'un neurone à l'erreur ainsi définie est calculée, et les poids des neurones sont modifiés en conséquence afin de réduire cette erreur. Le processus recommence ainsi jusqu'à ce qu'un taux d'erreur minimal acceptable soit atteint.

\subsubsection{Application du réseau de neurones}

Lee et al. [6] ont ainsi mis en place un contrôleur adaptatif s'appuyant sur un réseau de neurones qu'ils ont appliqué à un écoulement turbulent en canal pour un nombre de Reynolds $R_{c}=100$ défini à partir de la vitesse de frottement et de la demi-hauteur du canal, dans le but de réduire la traînée.

Le réseau est un réseau à une couche cachée, utilisant une somme pondérée des signaux d'entrée comme fonction de combinaison et une fonction tangente hyperbolique comme fonction de transfert. La couche cachée contient deux unités qui utilisent sept entrées correspondant à sept points distribués transversalement à l'écoulement entourant et incluant le point de calcul.

Les valeurs du cisaillement de paroi à chacun de ces points constituent les données d'entrée. La donnée de sortie est la valeur de la vitesse normale à la paroi que l'actionneur doit imposer au point de calcul.

$v_{j k}=W_{\mathrm{a}} \tanh \left(\sum_{i=-(N-1) / 2}^{(N-1) / 2} W_{i}\left(\frac{\partial w}{\partial y}\right)_{j, k+i}-W_{\mathrm{b}}\right)-W_{\mathrm{c}}$

où $j$ et $k$ sont des entiers indexant la position du point de calcul sur la grille de maillage, $v_{j k}$ la vitesse normale à la paroi au point de calcul de coordonnées $(j, k), w$ la vitesse selon la direction transversale, $y$ la direction normale à la paroi, $N$ le nombre de points nécessaires au calcul $(N=7$ dans l'étude de Lee et al. [6]) et $W_{a}, W_{b}$ et $W_{c}$ des nombres sans dimension représentant les poids introduits par la fonction de combinaison.

La phase d'apprentissage utilise un algorithme de rétro propagation. L'erreur $\varepsilon$ à minimiser est définie comme :

$$
\varepsilon=\frac{1}{2} \sum_{j} \sum_{k} \exp \left(\lambda\left|v_{j k}^{\mathrm{des}}\right|\right) *\left|v_{j k}^{\mathrm{des}}-v_{j k}\right|^{2}
$$

où $v_{j k}^{\text {des }}$ représente la vitesse normale de paroi à atteindre, au point de position $(j, k)$.

Le but est de limiter la traînée en modifiant le cisaillement à la paroi qui participe à la formation des tourbillons longitudinaux. La quantité cible n'est donc pas directement une valeur de la vitesse normale à la paroi, mais plutôt une valeur du cisaillement. Le terme $v_{j k}^{\text {des }}$ représente le contrôle à appliquer à l'écoulement afin d'obtenir un cisaillement de référence défini comme :

$$
\left.\frac{\partial w}{\partial y}\right|_{\text {desiree }}=\left.\eta \frac{\partial w}{\partial y}\right|_{t}
$$

avec $\eta<1$ un coefficient de proportionnalité qui règle la vitesse de convergence. Le paramètre $\eta$ a été pris égal à 0,85 . Lorsque le contrôle est appliqué, la traînée atteint rapidement $80 \%$ de sa valeur en écoulement non contrôlé.

Les modèles de réseaux artificiels présentent plusieurs propriétés intéressantes pour la simulation numérique de contrôle d'écoulement : ce sont des mailles massives d'éléments de calculs simples, ce qui permet d'utiliser des calculateurs parallèles beaucoup plus rapides que les ordinateurs classiques; ils sont également très robustes. Si quelques liaisons du réseau sont rompus, les performances n'en sont pas vraiment affectées; enfin, ils sont facilement adaptables. Ces propriétés font du réseau de neurones un choix judicieux pour modéliser en temps réel des structures évolutives.

L'intérêt du contrôle, et de la réduction de traînée en particulier, pour l'industrie automobile est double : réduire les émissions de gaz à effet de serre comme le $\mathrm{CO}_{2}$ et réduire les coûts de consommation du véhicule. Le coût énergétique du système de contrôle doit alors être suffisamment faible pour permettre un rendement énergétique positif. En ce sens, la stratégie de contrôle optimal est intéressante puisqu'elle cherche à optimiser le compromis entre la performance du contrôle et son coût.

\subsection{Schéma de contrôle optimal}

Le contrôle optimal prend en compte le coût de la perturbation apportée à l'écoulement en terme d'énergie. Une fonction coût, représentative de l'énergie apportée et de la performance du contrôle permet de s'assurer que celuici reste intéressant en terme de rentabilité. Ce système de contrôle nécessite donc un retour d'information de l'écoulement aval afin d'évaluer sa performance. C'est un système en boucle fermée.

Les équations régissant l'écoulement sont appelées équations d'état. Le critère (ou fonction coût) prend en compte un paramètre représentatif de l'état de l'écoulement : le frottement à la paroi [9], le cisaillement [10] ou le gradient de pression longitudinal $[4,9,10]$. Elle doit également tenir compte de l'énergie cinétique dépensée.

Pour un contrôle optimal, cette fonctionnelle sera minimisée sur un intervalle de temps, ce qui présente un coût considérable de calcul et de stockage. La méthode suboptimale introduite par Choi et al. [12] en 1993 constitue une bonne approximation à moindre coût. La fonctionnelle est en effet minimisée à chaque pas de temps. Une autre façon de contourner ce problème de coût est d'approcher les mouvements du fluide au voisinage de la paroi 
par extrapolation de quantités mesurées uniquement sur la paroi, ce qui rend en outre le contrôle utilisable sans capteur intrusif $[4,10]$.

\subsubsection{Procédure optimale}

Soit un écoulement caractérisé par un état $q=$ $\left(u_{1}, u_{2}, u_{3}, p\right)^{T}$ où $u_{1}, u_{2}$ et $u_{3}$ représentent les vitesses longitudinales, normales et transversales et $p$ la pression. Cet écoulement est soumis à une perturbation de contrôle $\phi$. La première étape est la discrétisation du système en temps et en espace. La deuxième étape concerne la fonctionnelle, qu'il faut définir en tenant compte de l'objectif du contrôle et de son coût. Soit $J(q, \phi)$ la fonctionnelle :

$$
J(q, \phi)=J_{\mathrm{P}}(q, \phi)+J_{\mathrm{C}}(\phi)
$$

où $J_{\mathrm{P}}(q, \phi)$ est une fonction représentative de la performance du contrôle, et $J_{\mathrm{C}}(\phi)$ une fonction représentative du coût du contrôle. Le choix de cette fonctionnelle est prépondérant pour la réussite du contrôle [10]. Enfin, la troisième étape concerne le calcul. Le but est de dégager la procédure qui permette de passer d'un pas de temps à l'autre.

Il s'agit d'abord de déterminer, puis de calculer la sensibilité de l'écoulement au contrôle à travers la dérivée de Fréchet, c'est-à-dire le gradient de la fonctionnelle en fonction de la perturbation $\frac{D J(\mathrm{q}, \phi)}{D \phi}$. Le calcul de ce gradient est délicat et passe par des inversions de matrices qui sont coûteuses en puissance de calcul, et donc en temps de réaction, d'où l'introduction d'un système adjoint. La détermination de la pression adjointe permet en effet de calculer le gradient de la fonctionnelle. Le contrôle est alors remis à jour à chaque pas de temps, en cherchant toujours à minimiser cette fonction coût, à travers un algorithme de type gradient ou plus profonde descente [5]. Pour des géométries simples où la séparation de variables est applicable, la décomposition de Fourier offre également une alternative intéressante [10].

L'étude de Choi et al. [11] sur le contrôle de l'écoulement autour d'une marche est présentée ici à titre d'exemple. L'objectif est de réduire la traînée par aspiration/soufflage. Une zone de contrôle $\Gamma_{\mathrm{c}}$ est définie au voisinage de l'arête (Fig. 9) et des capteurs mesurent le gradient adverse de fluctuation de pression au niveau du recollement du fluide, en aval de la marche $\left(\Gamma_{\mathrm{s}}\right.$ sur la Fig. 9).

\subsubsection{1 Équation d'état}

L'équation d'état se compose des équations de NavierStokes discrétisées avec un schéma de Crank-Nicholson pour les termes de pression et de viscosité et un schéma de Adams-Bashforth pour les termes de convection.

$$
\left\{\begin{array}{l}
u_{i}^{n+1}-\frac{\Delta t}{2 R e} \frac{\partial^{2} u_{i}^{n+1}}{\partial x_{j} \partial x_{j}}+\frac{\Delta t}{2} \frac{\partial p^{n+1}}{\partial x_{i}}+R^{n}=0 \\
\frac{\partial u_{j}^{n+1}}{\partial x_{j}}=0
\end{array}\right.
$$

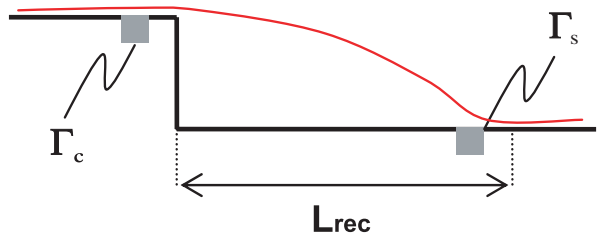

Fig. 9. Ecoulement autour d'une marche d'après Choi et al. [11]

avec $\left.u_{i}^{n+1}\right|_{w}=\phi\left(x_{3}\right) m_{i}$ sur la surface de contrôle $\Gamma_{\mathrm{c}}$ (avec $\left(m_{i}\right)$ la direction de soufflage) et nulle ailleurs. Les termes $x_{1}, x_{2}, x_{3}$ représentent respectivement les directions longitudinales, normales et transversales de l'écoulement, et $u_{1}, u_{2}, u_{3}$ les vitesses correspondantes. $R e$ représente le nombre de Reynolds et $\Delta t$ le pas de temps de la simulation numérique. $R$ contient les termes de convection, les termes explicites du gradient de pression et des termes de viscosité.

\subsubsection{Choix de la fonctionnelle}

Dans cette étude la fonctionnelle ne prend pas en compte le coût qui sera traité différemment. Le but est d'optimiser le gradient de pression tangent au niveau de la zone de recollement, c'est-à-dire sur $\Gamma_{\mathrm{s}}$. La fonctionnelle est donnée par :

$$
J(\phi)=\frac{1}{2} \int_{\Gamma_{\mathrm{S}}}\left(\frac{\partial p^{\prime}}{\partial x_{3}}\right)^{2} \mathrm{~d} x_{3} \mathrm{~d} x_{1}
$$

où $p^{\prime}$ représente une fluctuation de pression. Les équations (16) sont différenciées au sens de Fréchet. Soit :

$$
\begin{aligned}
& \left\{\begin{array}{l}
q_{i}^{n+1}-\frac{\Delta t}{2 R e} \frac{\partial^{2} q_{i}^{n+1}}{\partial x_{j} \partial x_{j}}+\frac{\Delta t}{2} \frac{\partial \rho^{n+1}}{\partial x_{i}}=0 \\
\frac{\partial q_{j}^{n+1}}{\partial x_{j}}=0
\end{array}\right. \\
& \text { avec }\left.q_{i}^{n+1}\right|_{w}=\tilde{\phi}\left(x_{3}\right) m_{i} \text { sur } \Gamma \mathrm{c} \\
& \left.q_{i}^{n+1}\right|_{w}=0 \text { sur } \Gamma \text { privé de } \Gamma \mathrm{c} .
\end{aligned}
$$

où $q$ et $\rho$ exprimant la sensibilité de la vitesse et de la pression à la perturbation. Le terme $R$ disparaît, ce qui se justifie par le fait que le terme $R$ au pas de temps $n$ est insensible à la perturbation au temps $n+1$. La résolution passe alors par la méthode des équations adjointes.

\subsubsection{Problème adjoint}

Soit le système adjoint suivant $\left(\eta_{i}, \pi\right)$ avec cette fois :

$$
\begin{aligned}
& \left\{\begin{array}{l}
\eta_{i}^{n+1}-\frac{\Delta t}{2 R e} \frac{\partial^{2} \eta_{i}^{n+1}}{\partial x_{j} \partial x_{j}}+\frac{\Delta t}{2} \frac{\partial \pi^{n+1}}{\partial x_{i}}=0 \\
\frac{\partial \eta_{j}^{n+1}}{\partial x_{j}}=0
\end{array}\right. \\
& \text { où }\left.\eta_{i}^{n+1}\right|_{w}=\delta\left(\chi_{3}\right) \text { sur } \Gamma_{\mathrm{c}} \\
& \left.\eta_{i}^{n+1}\right|_{w}=0 \text { sur } \Gamma \text { privé de } \Gamma_{\mathrm{c}} .
\end{aligned}
$$


Les systèmes (18) et (19) sont les mêmes, aux conditions limites près. C'est un problème auto-adjoint. Ce système est résolu numériquement en considérant un Dirac en $\Gamma_{\mathrm{c}}$. Le couple $(\eta i, \pi)$ ainsi obtenu peut être relié au couple $(q i, \rho)$ par un produit de convolution.

$$
\left\{\begin{aligned}
q_{i}\left(x_{1}, x_{2}, x_{3}\right) & =\int_{0}^{L} \eta_{i}\left(x_{1}, x_{2}, x_{3}-\varsigma\right) \tilde{\phi}(\varsigma) \mathrm{d} \varsigma \\
\rho\left(x_{1}, x_{2}, x_{3}\right) & =\int_{0}^{L} \pi\left(x_{1}, x_{2}, x_{3}-\varsigma\right) \tilde{\phi}(\varsigma) \mathrm{d} \varsigma
\end{aligned}\right.
$$

\subsubsection{Détermination du contrôle optimal}

La sensibilité de $J$ à $\phi$ est donnée par :

$$
\frac{D J(\phi)}{D \phi}=\frac{1}{2} \int_{\Gamma_{S}}\left(\frac{\partial p^{\prime}(\varsigma)}{\partial \zeta}\right)\left(\frac{\partial \pi^{\prime}\left(\zeta-x_{3}\right)}{\partial \zeta}\right) \mathrm{d} \zeta
$$

La perturbation qui maximise $J$ est alors déterminée par un algorithme de type gradient indexé par $k$ :

$$
\phi_{k+1}^{n+1}=\phi_{k}^{n+1}+\beta\left(\frac{D J}{D \phi^{n+1}}\left(\phi_{k}^{n+1}\right)\right)
$$

où $\beta$ représente le paramètre de déplacement de l'algorithme.

Soit par développement limité

$$
\begin{aligned}
J\left(\phi_{k+1}^{n+1}\right) & \approx J\left(\phi_{k}^{n+1}\right)+\frac{D J}{D \phi^{n+1}}\left(\phi_{k}^{n+1}\right)\left(\phi_{k+1}^{n+1}-\phi_{k}^{n+1}\right) \\
& \approx J\left(\phi_{k}^{n+1}\right)+\beta \mid \frac{D J}{D \phi^{n+1}}\left(\phi_{k}^{n+1}\right)
\end{aligned}
$$

La relation (23) est itérée sur $k$ jusqu'à la convergence. Ce genre de contrôle, bien que très rigoureux, est donc extrêmement coûteux en terme de temps de calcul, et donc peu adapté à des projets de systèmes embarqués. Lorsque la relation (23) a convergé, la perturbation apportée à l'écoulement par le contrôleur est optimale. Elle minimise le gradient de la fonctionnelle définie en (21). L'expression de la perturbation optimale est alors donnée par (24).

$$
\phi^{n+1}\left(x_{3}\right)=\beta \int_{\Gamma_{S}}\left(\frac{\partial p^{\prime}(\varsigma)}{\partial \zeta}\right)\left(\frac{\partial \pi^{\prime}\left(\zeta-x_{3}\right)}{\partial \zeta}\right) \mathrm{d} \zeta
$$

Cette perturbation appliquée à l'écoulement au temps $t=(n+1) \Delta t$ où $\Delta t$ représente le pas de temps physique de la simulation assure le meilleur compromis entre performance et coût du contrôle.

Le coût est envisagé à travers le paramètre $\beta$ qui est déterminé de façon à avoir une valeur moyenne quadratique de $\phi$ constante et qui peut éventuellement être nulle. Cependant, cette expression n'étant pas très commode, et toujours pour réduire les contraintes de coût en vue d'un système embarqué, un contrôle en boucle ouverte va être mis en place à partir de l'analyse de l'évolution temporelle du contrôle.

\subsubsection{Application de la stratégie optimale}

Lee et al. [10] ont développé un contrôle suboptimal ne nécessitant que des informations de paroi (pression et vitesse) sur un écoulement turbulent en canal à $R e=110$. Le code utilisé est le code spectral développé par Kim et al. [13]. L'intégration de la fonctionnelle de coût se fait sur la surface de la paroi pendant une durée élémentaire $\Delta t$ correspondant typiquement au pas de temps du code de résolution numérique. Le contrôle optimal est déterminé dans l'espace de Fourier, et défini par une intégrale de convolution qui nécessite les données de tout l'écoulement. Une simulation numérique a donc été conduite sur la transformée inverse, afin de proposer une approximation pondérée de ce contrôle ne nécessitant que des données de paroi. En travaillant sur le gradient de pression transversale à la paroi, la réduction de traînée obtenue est de $16 \%$. Elle est de $22 \%$ si la fonctionnelle inclut le flux de cisaillement à la paroi.

Choi et al. [11] se sont intéressés à un autre problème redondant en mécanique des fluides : le mélange en aval d'une marche, en écoulement turbulent. Le but est d'améliorer ce mélange, notamment en diminuant la longueur de recollement $L$. Physiquement, le contrôle se fera par soufflage/aspiration localisé sur l'arête de la marche sur une épaisseur représentant le dixième de la hauteur de la marche. Le jet est incliné à 45 degrés. Numériquement, le gradient tangent de pression, en partie responsable du mélange est inclus dans la fonctionnelle de coût. La simulation se fait par LES (Large-Eddy Simulation) à $R e=5100$. Le domaine de calcul est maillé par une grille $(151,56,64)$ non uniforme, resserrée vers la marche. Le schéma numérique est une variante de celui présenté par Kim et al. [14] où les termes de convection et de diffusion normaux à la paroi sont modélisés par un schéma de Crank-Nicholson, les autres termes utilisant un schéma de Runge-Kutta du $3^{\mathrm{e}}$ ordre. Dans un premier temps, un contrôle en boucle ouverte, sinusoïdal, dont la fréquence est paramétrée par le nombre de Strouhal, permet de ramener la longueur de recollement à 1,2 fois la hauteur de la marche, soit une réduction de 19,4\% par rapport à l'écoulement non contrôlé. Le contrôle optimal est ensuite appliqué et entraîne une réduction de $27,3 \%$ de la longueur de recollement.

La stratégie de commande optimale permet d'obtenir des résultats intéressants en terme de réduction de traînée [10] et de propriété de mélange [11]. Elle assure de plus un gain énergétique en imposant un critère de coût. Cependant, la quantité importante de calculs nécessaire à sa mise en place ne permet pas aujourd'hui d'envisager son utilisation en système embarqué. Un système adjoint et divers algorithmes de type gradient sont en effet résolus à chaque pas de temps la réactivité du système est donc trop lente pour des applications automobiles. Le temps de calcul est doublé lorsque le contrôle optimal est mis en place dans l'étude numérique de Creusé [4]. La mise en place d'une stratégie de contrôle optimal doit donc inclure des schémas de simplification permettant de limiter la taille des calculs nécessaires. 


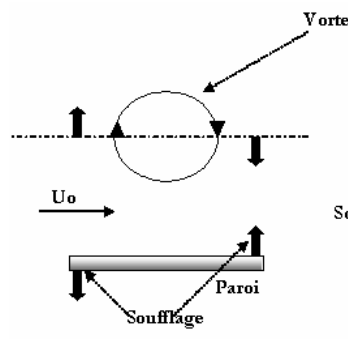

(a)

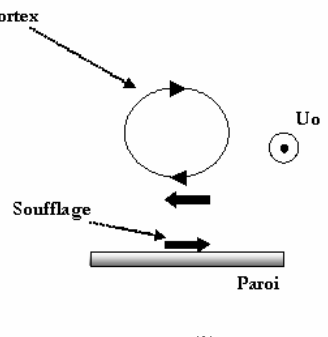

(b)
Fig. 10. Schéma de contrôle d'après Choi et al. [12] (a) contrôle sur la vitesse normale (b) contrôle sur la vitesse transversale.

\section{Simplification des schémas}

\subsection{Schéma de contrôle basé sur des considérations physiques}

Dans des situations où la structure de l'écoulement est bien connue, l'analyse de la formation des structures cohérentes impliquées par exemple dans l'augmentation de la traînée peut guider la conception d'un schéma de contrôle. Ce type de conception est cependant assez limité. Il ne s'applique pas par exemple à des écoulements où la turbulence est pleinement développée. Néanmoins, quand elle est applicable, cette technique permet une étude amont qui simplifie nettement la complexité du système à contrôler, et donc la mise en place de boucle fermée de type adaptative ou optimale.

Les études réalisées dans ce sens traitent généralement du contrôle des tourbillons de proche paroi. Dans ce cas, l'objectif consiste à éloigner de la paroi la région dans laquelle se développent les contraintes tangentielles visqueuses participant à la traînée aérodynamique. Plus la distance à la paroi est grande, plus le contrôle est performant [12]. Choi et al. [12] ont ainsi limité l'effet des vortex dans un écoulement pleinement turbulent en canal. La vitesse du fluide injecté par l'actionneur, imposée à la paroi au niveau des capteurs est ici définie comme l'opposé de la vitesse détectée sur un plan parallèle à la paroi au voisinage de celle-ci pour le contrôle de tourbillons transversaux (Fig. 10a) ou longitudinaux (Fig. 10b).

Choi et al. [12] obtiennent ainsi grâce à une stratégie suboptimale simplifiée une réduction de traînée de $25 \%$ en travaillant sur la vitesse radiale (Fig. 10a) et $30 \%$ avec la vitesse transversale (Fig. 10b). Les gains chutent à $10 \%$ en imposant une vitesse longitudinale. Ce schéma est donc très performant, mais inutilisable expérimentalement puisqu'il fait appel à des capteurs intrusifs.

Koumoutsakos [15] développe également un schéma de contrôle basé sur le mécanisme physique de formation de la vorticité à la paroi. Il étudie l'interaction entre un dipôle de vortex et une paroi. Le flux de vorticité à la paroi est calculé à partir de la mesure du gradient de pression de paroi. Un schéma de contrôle simple en boucle fermée de type adaptatif permet alors de déterminer la distribution de vitesse de contrôle à appliquer à la paroi afin d'obtenir le flux de vorticité désiré. La structure des tourbillons de l'écoulement est fortement perturbée par la présence des vortex induits par le soufflage de paroi, et la traînée perd $40 \%$ de sa valeur. Il apparaît que les résultats sont similaires à ceux de Choi et al. [12] en n'utilisant que des variables mesurées à la paroi.

Creusé [4] applique le même contrôle à l'écoulement décrit dans le la section 2.1. L'influence du nombre de capteurs et d'actionneurs, et leurs localisations est étudiée. La configuration la plus performante utilise 8 capteurs et 8 actionneurs. Le coefficient de récupération de pression est alors de 85,9\%. En se basant sur des arguments physiques, Creusé obtient donc de meilleurs résultats que la stratégie adaptative $(84,71 \%$ en 2.2 .2$)$ ou que le contrôle en boucle fermée simple $(84,08 \%$ en 2.1$)$. Les actions les plus fortes sont visiblement exercées par les actionneurs les plus en amont ce qui semble indiquer que ces derniers ont une influence prépondérante sur l'écoulement. L'écoulement est plus sensible aux perturbations amont.

Pour des systèmes plus complexes qui font intervenir la turbulence, la structure tourbillonnaire de l'écoulement est difficilement prévisible, et un deuxième schéma de simplification est préconisé, basé cette fois sur une décomposition en mode dynamique.

\subsection{Schéma de contrôle basé sur un système dynamique}

Les écoulements turbulents sont connus pour avoir un nombre très important de degrés de liberté dû aux nombreuses échelles spatiales et temporelles qui s'y côtoient et interagissent. L'analyse de tels écoulements est alors très difficile, voire impossible, même pour de faibles nombres de Reynolds [16].

La théorie des systèmes dynamiques non-linéaires permet cependant de décomposer la turbulence en un petit nombre de modes représentatifs dont les comportements dynamiques sont analysés afin de déterminer la meilleure loi de contrôle. Ces outils ont prouvé leur utilité dans l'analyse et l'interprétation de la turbulence [17]. La majeure partie de ces travaux est basée sur la décomposition orthogonale propre (POD) introduite par Lumley en 1967, qui propose une procédure dans la recherche d'une base de décomposition modale appropriée. Les statistiques du second ordre sont alors assez bien représentées par un jeu de fonctions propres. Cette décomposition est néanmoins limitée par son caractère linéaire [18].

Aubry et al. [17] représentent ainsi les équations différentielles d'une couche limite turbulente, de dimension infinie a priori, à l'aide d'un système d'équations différentielles de dimension 10. Ces équations décrivent le comportement dynamique des tourbillons mais font apparaître un comportement déterministe, périodique en opposition avec la nature stochastique de l'écoulement turbulent. Berkooz et al. [19] reprennent ce modèle en découplant les tourbillons longitudinaux et transversaux. Leurs résultats laissent entendre que le caractère déterministe des résultats de Aubry n'est pas dû uniquement à la modélisation utilisée mais à un comportement propre à l'écoulement. 


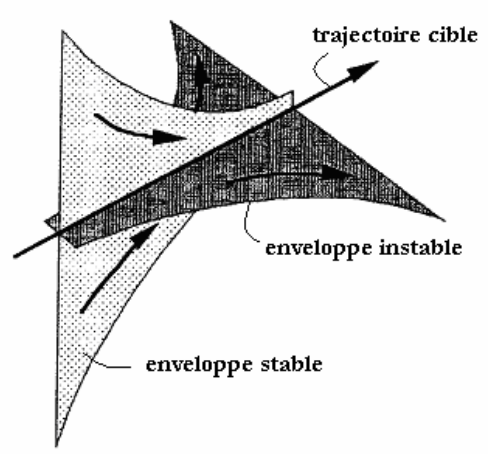

Fig. 11. Schéma de la méthode OGY d'après Gad-El-Hak [20].

Phénomènes déterministe et chaotique se côtoient au sein d'un écoulement turbulent. C'est ce constat qui pousse de nombreux scientifiques à chercher à contrôler les écoulements chaotiques [20]. Keefe [21,22] utilise ainsi la méthode OGY (Ott, Grebogy et Yorke en 1990) afin de stabiliser la turbulence. Selon ces auteurs, l'état de la turbulence peut être représenté schématiquement par l'intersection d'une enveloppe stable et d'une enveloppe instable (Fig. 11). Les perturbations induites par le contrôle amènent l'écoulement dans un état de turbulence correspondant donc également à une telle intersection. Tout état se trouvant sur l'enveloppe stable va alors naturellement converger vers cet état en l'absence de bruit. L'idée est alors de se servir de cette convergence naturelle en contrôlant ponctuellement la distance séparant le système de l'enveloppe stable. Cela permet ainsi de limiter l'énergie de contrôle. Keefe obtient de 60 à $80 \%$ de réduction sur la traînée d'un écoulement turbulent en canal en utilisant cette méthode.

Les simplifications de type POD sont ainsi souvent utilisées en prétraitement d'une stratégie de contrôle optimal. Elles permettent de limiter le nombre de degrés de liberté du système et donc de simplifier la mise en place du contrôle.

\section{Discussion}

Les attentes du secteur automobile portent sur le coût, la performance en terme de réduction de traînée et d'adaptabilité, et la faisabilité du système de contrôle. Le schéma développé doit permettre au contrôle une réduction de l'ordre de $20 \%$ de la traînée aérodynamique. Il doit en outre être réalisable industriellement, être adaptable à différentes configurations d'écoulement et satisfaire des contraintes de coût en énergie.

Un état de l'art des différentes stratégies numériques de contrôle est présenté dans ce rapport. Les stratégies sont classées selon un organigramme très pédagogique proposé initialement par Gad-El-Hak [1]. La stratégie de contrôle optimale est la plus rigoureuse [1], et la plus proche des considérations mathématiques. Son coût en calcul, même après simplification, ne permet cependant pas d'envisager des simulations numériques en 3D sur un véhicule. Dans le cadre du contrôle des décollements, une telle stratégie est donc caduque à l'heure actuelle, les phénomènes tourbillonnaires issus du décollement étant essentiellement tridimensionnels. Elle est cependant amenée à se développer avec les possibilités de calcul numérique, et pourrait représenter la voie la plus intéressante à long terme.

La stratégie adaptative présente également plusieurs caractéristiques intéressantes. Elle ne nécessite pas de connaissances a priori de l'écoulement et peut s'adapter à l'évolution du système, ce qui rejoint les attentes du secteur automobile. Elle est de plus assez robuste aux perturbations extérieures. Néanmoins, elle est également assez lourde en calcul, ce qui peut rendre le temps de réaction trop lent pour être vraiment réactif. Les informations manquent dans la bibliographie pour estimer tous ces coûts en calcul et temps de réaction, mais une étude plus poussée pourrait être intéressante pour les systèmes futurs. L'utilisation de réseau de neurones semble être notamment une voie à explorer.

En pratique, ces stratégies sont couplées. La procédure de conception d'un contrôle peut ainsi s'articuler autour de 3 étapes. Une première étude dynamique, basée sur des considérations physiques analyse le problème et détermine une loi théorique de commande. Une stratégie de contrôle optimal ou adaptatif développe ensuite un algorithme autour de cette loi de commande afin de l'optimiser. Enfin, l'évolution temporelle du forçage obtenu est analysée et modélisée par une loi de commande simple en boucle ouverte [10-12].

Une telle loi de commande, en boucle ouverte, ne permet cependant pas à l'actionneur d'être vraiment réactif. Elle est essentiellement performante pour des écoulements stationnaires, ce qui n'est généralement pas le cas en automobile. Cet état de fait induit deux perspectives : identifier les principales configurations d'écoulement rencontrées et développer un système performant pour chacune d'elles ou développer un système adaptable. Ce dernier est inévitablement plus lent en terme de temps de réponse. Les réseaux de neurones appliqués aux schémas adaptatifs peuvent alors constituer une alternative intéressante.

\section{Références}

[1] M. Gad-el-Hak, Modern developments in flow control, Appl. Mech. Rev. 9 (1996) 365-379

[2] P. Moin, T. Bewley, Feedback control of turbulence, Appl. Mech. Rev. 47 (1994)

[3] S. Tardu, contrôle actif de la turbulence pariétale, Actes du XV Congrès Français de Mécanique, Nancy, 3-7 septembre 2001

[4] E. Creusé, Simulation et contrôle actif d'écoulements compressibles, Thèse de doctorat, Université de Bordeaux 1, 2000

[5] F. Michaud, Méthodes adaptatives pour le signal, Éditions Hermes Paris, ISBN 978-2-86601-310-3 (1992)

[6] C. Lee, J. Kim, D. Babcock, R. Goodman, Application of neural networks to turbulence control for drag reduction, Phys. Fluids 9 (1997) 170-1747 
[7] O. Sarzeaud, Les Réseaux de Neurones : Contributions à une théorie, OUEST Éditions ISBN 29082619441995

[8] M. Jacek Zurada, Introduction to Artificial Neural Systems, West Publishing Company ISBN 053495460X 1992

[9] T. Bewley, P. Moin, R. Teman, DNS-based predictive control of turbulence: an optimal benchmark for feedback algorithms, J. Fluid Mech. 447 (2001) 179-225

[10] C. Lee, J. Kim, H. Choi, Suboptimal control of turbulent channel flow for drag reduction, J. Fluid Mech. 358 (1998) $25-258$

[11] H. Choi, S. Kang, Suboptimal feedback control of turbulent flow over a backward-facing step, J. Fluid Mech. 463 (2001) 201-227

[12] H. Choi, P. Moin, J. Kim, Active turbulence control for drag reduction in wall-bounded flows, J. Fluid Mech. 262 (1993) $75-110$

[13] J. Kim, P. Moin, R. Moser, Turbulence statistics in fully developed channel flow at low Reynolds number, J. Fluid Mech. 177 (1987) 133

[14] J. Kim, P. Moin, Application of a fractionnal-step method to incompressible Navier-Stokes equations, J. Comp. Phys. 59 (1985) 308-323
[15] P. Koumoutsakos, Active control of vortex-wall interaction, Phys. Fluids 9 (1997) 3808-3816

[16] L. Keefe, P. Moin, J. Kim, The Dimension of attractors underlying periodic turbulent Poiseuille flow, J. Fluid Mech. 242 (1992) 1-29

[17] N. Aubry, P. Holmes, J. Lumley, The dynamic of coherent structures in the wall region of a turbulent boundary layer, J. Fluid Mech. 192 (1988) 115-173

[18] G. Berkooz, P. Holmes, J. Lumley, The proper orthogonal decomposition in the analysisof turbulent flow, Ann. Rev. Fluid Mech. 25 (1993) 539-575

[19] G. Berkooz, P. Holmes, J. Lumley, Intermittent dynamics in simple models of the turbulent wall layers, J. Fluid Mech. 230 (1991) 75-95

[20] M. Gad-El-Hak, Flow control: the future, J. Aircraft 38 (2001)

[21] L. Keefe, Drag reduction in channel flow using non-linear control, AIAA Paper (1993) 93-3279

[22] L. Keefe, Two non linear control schemes contrasted on a hydrodynamic like model, Phys. Fluids A 5 (1993) 931947 